\title{
Improving the efficiency of the milling process by reducing the oscillation of the technological system
}

\author{
Alexander M. Kozlov ${ }^{1, *}$, Evgeny $V$. Kiryuschenko ${ }^{2}$, and Andrey A. Kozlov ${ }^{1}$ \\ ${ }^{1}$ Lipetsk State Technical University, 398600, 30, Moskovskaya Str., Lipetsk, Russia \\ ${ }^{2}$ PJ-SCo. Novolipetsky Metallurgical Complex, 398040 2, Metallurgists' Square, Lipetsk, Russia
}

\begin{abstract}
The paper reports the dynamics of the technological system during the milling of complex parts, and the investigation of possibly efficiency increase of the cutting process by creating conditions for system self-adjustment, what will cause the reduction of the common level of its oscillation.
\end{abstract}

\section{Introduction}

The modern technology of multiaxis milling allows manufacturing parts with various forms, but the productivity of such a process has a number of limitations. Such limitations may be requirements to accuracy assurance in a form, parameters of worked surface roughness, and in some cases, - rigidity of a tool and a technological system on the whole $[1,2]$.

The impossibility to increase rigidity considerably causes oscillations when cutting which affects in a negative way upon quality and productivity of processing.

As a rule there is an approach to the problem of milling efficiency increase from the standpoint of vibrations decrease. A basic problem at the development of methods for milling efficiency increase is an adequate presentation of basic regularities in the excitation of forced oscillations and self-oscillations as factors defining machining accuracy $[3,4,5]$.

\section{System investigation}

A technological system is considered as a mechanical oscillation system with the contour of some masses connected between each other with elastic and dissipative elements (Fig. 1).

The vector equation of the motion of the $i$-th mass:

$$
m_{i} \cdot \bar{a}_{i}=\bar{F}_{i}+\bar{P}_{i}-\bar{F}_{i+1}
$$

$P_{i}$ - external force influence upon the $i$-th mass, $\mathrm{H}$; $F_{i}$ - force causing elastic damping on the $i$-th unit, $\mathrm{H} ; m$ - mass of the unit, $\mathrm{kg} ; a$ - unit acceleration, $\mathrm{mm} / \mathrm{s}^{2}$.

$$
F_{i}=C_{i} \cdot\left(X_{i-1}^{\prime}-X_{i}^{\prime}\right)+h_{i} \cdot\left(v_{i+1}-v_{i}\right)
$$

$F u_{i}=-m_{i} \cdot a_{i}$ - inertial force on the $i$-th unit, $\mathrm{H}$;

$X^{\prime}{ }_{i}$ - vibrodisplacement of the $i$-th mass, mm; $v_{i},-$ vibrovelocity of the $i$-th mass, $\mathrm{mm} / \mathrm{s}$. [6]

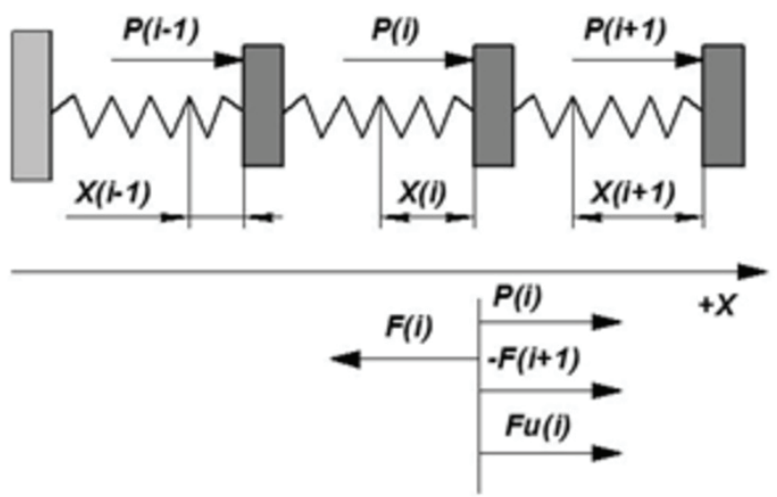

At stretching

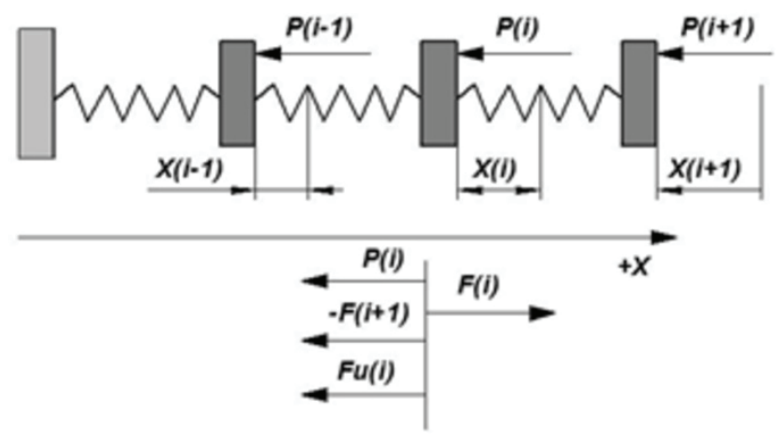

At pressing

Fig. 1. Design scheme of an elastic system contour.

Vibration velocity is a velocity of an oscillating mass displacement. From the standpoint of vibro-diagnostics it is a universal parameter which allows comparing different oscillating processes. With its aid it is possible to determine a correlation of an oscillating process with cutting forces changes.

It is well-known [7, 8, 9] that when milling the primary oscillations are formed with secondary resonant

kam-48@yandex.ru 
forced oscillations. In such a way, the vibrations of the technological system are a sum of some oscillating processes. Hence, on the basis of the analysis of experimental data there is obtained a dependence describing changes in vibro-velocity of a milling machine spindle:

$$
v=A_{1} \cdot e^{-0,1 S} \cos \left(f_{1} \cdot S+\varphi_{11}\right)+A_{2} \cdot e^{-0,1 n} \sin \left(f_{2} \cdot n+\varphi_{12}\right)+v_{0}
$$

$v$ - vibrovelocity in the system, $\mathrm{mm} / \mathrm{s} ; A_{1}$ and $A_{2}-$ amplitudes of corresponding oscillating processes, $\mathrm{mm}$; $S$ - feed, $\mathrm{mm} / \mathrm{min} ; n$ - rotation frequency, $\mathrm{rpm} ; f-$ frequency of corresponding oscillations, $\mathrm{Hz} ; \varphi-$ difference of oscillation phases in the corresponding direction, rad; $v_{0}$ - vibrovelocity out of a cutting area, $\mathrm{mm} / \mathrm{s}$.

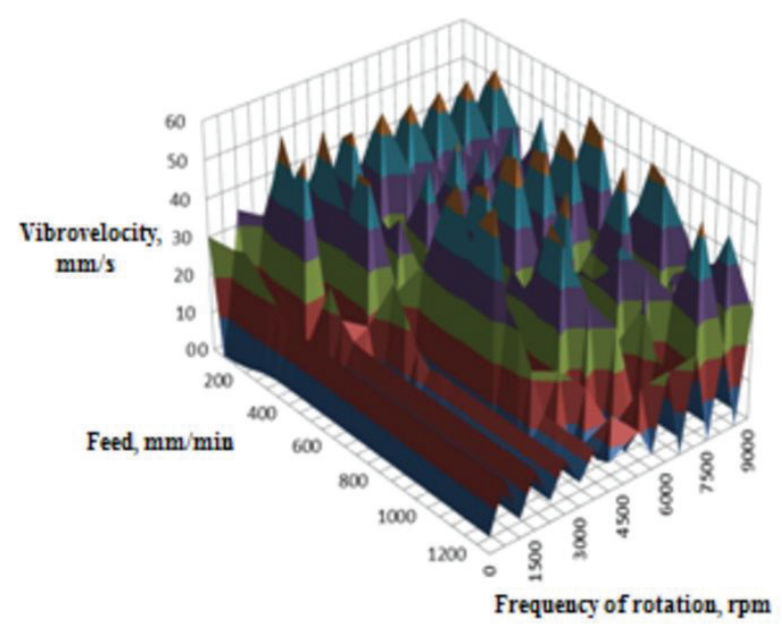

Fig. 2. Machining parameter impact upon vibrovelocity.

The peculiarity of a milling process is an interrupted character of cutting which may be characterized by lag time. Lag time corresponds to the value of phase difference this is the same time interval, but measured in a phase (id est, in the angles of the phase):

$$
\varphi_{21 x}=\omega_{x} \cdot \tau_{21}=\omega_{x} \cdot(k+i) \cdot T_{x}=(k+i) \cdot 2 \pi
$$

$\varphi_{21 x}$ - difference of phase oscillations in the system on $\mathrm{X}$ axis during two adjacent teeth motion through the given point in the area, rad; $\mathrm{rad} / \mathrm{s}$.

$\omega_{x}-$ angular frequency of oscillations on $\mathrm{X}$ axis,

If we consider a trace on the surface of cutting as a driving force with the frequency $p$, then the phase of forced oscillations of the system must lag behind the phase of a disturbing force for the value:

$$
2 \pi \cdot i_{p}=-\arctan \frac{2 \cdot \varepsilon \cdot \gamma}{1-\gamma^{2}}
$$

$\varepsilon$ - dimensionless factor of attenuation; $\gamma$ - ratio of the frequency of the driving force $p$ to the own frequency $f_{x}$.

During the analysis of vibrovelocity changes (Fig.2) on the basis of equations (2) and (3) there is observed a soft excitation of regenerative oscillations. It appears from this that the system steady at cutting on a trace but with a small coefficient of rigidity may become unsteady at cutting along a trace when a rigidity coefficient will exceed a certain value. At the equality of own and driving frequencies the phase displacement does not depend upon $\varepsilon$ may be equal only to $-\pi / 2$. Strictly speaking, this value of the phase displacement corresponds to conditions of resonance occurrence [10].

In such a way, the increase of oscillations level at disturbances on a trail is determined, in the first place, by resonance and near-resonance phenomena caused by a coincidence of the own frequency of the system with the frequency of the wave on the surface of cutting. The results obtained have also shown that the parameter affecting the level of oscillations is a phase displacement at the initial moment of trail cutting. At that a minimum level of oscillations occurs at the initial displacement equal to $\pi / 2$, and a maximum one - at $-\pi / 2$. At that, if the initial displacement of phases differs from $-\pi / 2$, then a part of oscillations energy is consumed for the system tuning, and oscillations are attenuated rather quickly. If the initial displacement of phases is equal to $-\pi / 2$ or close to this value, then the system is set up immediately to the essential displacement of phases and in the system arise the established oscillations. In consequence of the existence of friction in the system such oscillations are damped gradually and damping time depends upon the initial displacement of phases.

\section{Oscillations damping}

It is possible to eliminate resonant oscillations with large amplitudes, if a damping device is introduced into the structure of metal-cutting equipment. In this case a damping device is an additional body with the mass of $m_{2}$ connected with the object through a spring with the rigidity of $c_{2}$ and a damper with the coefficient of viscous resistance, $h$.

In the presence of a damper a complete oscillations damping becomes impossible as it affects in addition a technological system. But a damper allows obtaining the limited amplitude of system oscillations in all the frequency range at an efficient selection of damper parameters (Fig. 3). This phenomenon may be called a self-tuning of the oscillation system $[11,12]$.

Differential equations of the system with a damper become:

$$
\begin{gathered}
m_{1} \ddot{x}_{1}+\dot{h} \dot{x_{1}}+\left(c_{1}+c_{2}\right) x_{1}-\dot{h} \dot{x_{2}}-c_{2} x_{2}=F_{i} \\
m_{2} \ddot{x_{2}}-h \dot{x_{1}}-c_{2} x_{1}+h \dot{x_{2}}+c_{2} x_{2}=0
\end{gathered}
$$

$m_{1}-$ system mass, $\mathrm{kg} ; m_{2}-$ damper mass, $\mathrm{kg} ; c_{1}-$ system rigidity, $c_{2}$ - damper rigidity, $h$ - coefficient of viscous resistance.

Let us add to these equations some dimensionless parameters: $\beta, \eta, \delta$.

$$
\beta=\frac{m_{2}}{m_{1}}, \quad \delta=\frac{\sqrt{c_{2} / m_{2}}}{\omega_{0}}, \eta=\frac{h}{2 m_{2} \omega_{0}},
$$


$\omega_{0}$ - eigen frequency of machine oscillations, $1 / \mathrm{s} ; n_{2}$ - partial frequency of the damper, $1 / \mathrm{s}$.

Then, after transforms we obtain an expression for the dimensionless amplitude of damper oscillations:

$$
A_{1}=\sqrt{\frac{\left(\delta^{2}-\gamma^{2}\right)^{2}+4 \eta^{2} \gamma^{2}}{\left[\left(1-\gamma^{2}\right)\left(\delta^{2}-\gamma^{2}\right)-\beta \delta^{2} \gamma^{2}\right]^{2}+4 \eta^{2} \gamma^{2}\left(1-\gamma^{2}-\beta \gamma^{2}\right)^{2}}}
$$

The expression obtained allows calculating the amplitude of oscillations for a damping device for the achievement by a technological system a self-tuning state.

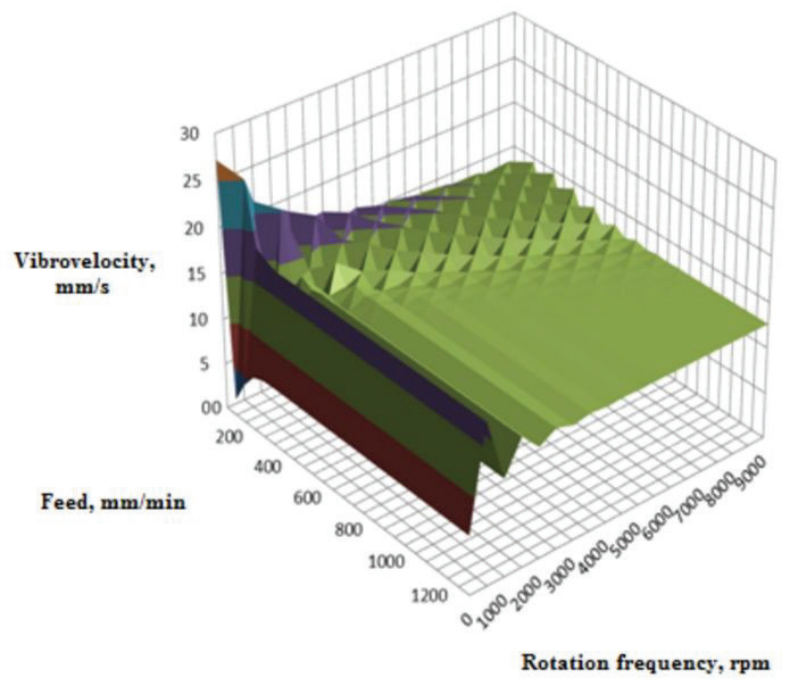

Fig. 3. Theoretical limitation of values of the spindle vibrovelocity in milling equipment at damping.

The expression obtained allows calculating the amplitude of oscillations for a damping device for the achievement by a technological system a self-tuning state.

In such a way, the more power for tuning is consumed, the less it becomes for oscillations excitations. To reduce a regenerative effect it is possible by means of the system tuning from resonance with a trail. If a wave period on the surface of cutting does not coincide with the period of eigen oscillations, then resonance will not appear and the level of oscillations will be considerably lesser.

\section{Conclusion}

In such a way, at the phase displacement for $-\pi / 2$ occurs a system self-tuning as a result of which a part of oscillation processes is consumed and a general level of oscillations will drop. The more energy is consumed for tuning, the lesser it is for oscillations excitation. It results from this that one of the efficient ways for regenerative oscillations damping may be the formation of such conditions for its operation at which it should be necessary for it to carry out constantly such a retuning. It may be achieved with the aid of a special damping device located on bearings of machine spiral gears. At that if the period of the wave on the surface of cutting does not coincide with the period of eigen oscillations, then resonance will not appear and the level of oscillations will be much lesser.

\section{References}

1. R. A. Radulescu, General Cutting Process Model for High Speed Machining Dynamic and Thermal Considerations: PhD Thesis, University of Illinois at Urbana-Champaign, p. 257 (1993)

2. V.M. Svinin, Metal Machining, 3(28), 28-30 (2005)

3. A.M. Kozlov, E.V. Kiryushchenko, Reference Book. Engineering Journal, 1, 30-34 (2013)

4. A.M. Kozlov, E.V. Kiryushchenko, Fundamental and Applied Problems in Engineering and Technology, 3, 65-73 (2012)

5. S. Sastry, S. G. Kapoor, R. E. DeVor, G.E. Dullerud, ASME J. Eng. Indus., 123, 753-756 (2001)

6. A.M. Kozlov, E.V. Kiryushchenko, S.F. Kuznetsov, Fundamental and Applied Problems in Engineering and Technology, 5, 91-97 (2014)

7. Yu.I. Gorodetsky, S.N. Strebulyaev, Yu.E. Maiorova Bulletin of Scientific Technical Development, National Technological Group, 9(25), 91-95 (2005)

8. S.A. Vasin, Prediction of Tool Vibration Resistance at Turning and Milling (Mechanical Engineering Moscow, 2006), 384

9. A.M. Kozlov, G.E. Malyutin, Bulletin of Mechanical Engineering, 12, 71-75, 2014

10. A.M. Kozlov, E.V. Kiryushchenko, S.F. Kuznetsov Current Problems in Mechanical Engineering: Proceedings of the VII-th Inter. Scientific-Tech. Conf. (Tomsk Polytechnic University, Tomsk, 2013), 295299

11. A.M. Kozlov, G.E. Malyutin, Science Intensive Technologies in Mechanical Engineering, 14-18 (2013)

12. A.M. Kozlov, E.V. Kiryushchenko, Current Fundamental and Applied Investigations, 1(4) 32-38, (2012) 